\title{
A Prospective Cohort Study of Factors Associated with Empiric Antibiotic De-escalation in Neonates Suspected with Early Onset Sepsis (EOS)
}

\author{
Nazedah Ain Ibrahim ${ }^{1} \cdot$ Mohd Makmor Bakry $^{1}$ - Nurul Ain Mohd Tahir ${ }^{1} \cdot$ Nur Rashidah Mohd Zaini $^{2}$. \\ Noraida Mohamed Shah ${ }^{1}$
}

Published online: 18 March 2020

(c) Springer Nature Switzerland AG 2020

\begin{abstract}
Background Prolonged empiric antibiotic use, resulting from diagnostic uncertainties, in suspected early onset sepsis (EOS) cases constitutes a significant problem. Unnecessary antibiotic use increases the risk of antibiotic resistance. Furthermore, prolonged antibiotic use increases the risk of mortality and morbidity in neonates. Proactive measures including empiric antibiotic de-escalation are crucial to overcome these problems.

Methods This was a prospective cohort study conducted in the neonatal intensive care units of two public hospitals in Malaysia. Neonates with a gestational age greater than 34 weeks who were started on empiric antibiotics within $72 \mathrm{~h}$ of life were screened. The data were then stratified according to de-escalation and non-de-escalation practices, where de-escalation practice was defined as narrowing down or discontinuation of empiric antibiotic within $72 \mathrm{~h}$ of treatment.

Results A total of 1045 neonates were screened, and 429 were included. The neonates were then divided based on de-escalation $(n=207)$ and non-de-escalation $(n=222)$ practices. Neonates under non-de-escalation practices showed significantly longer durations of antibiotic use compared to those under de-escalation practices $(p<0.05)$, with no difference in treatment outcomes. Five factors were found to be associated with de-escalation of antibiotics. They are cesarean section delivery, exposure to antenatal steroids, nil history of maternal pyrexia, absence of meconium-stained amniotic fluid, and normal C-reactive protein $\leq 0.5 \mathrm{mg} / \mathrm{dL}(p<0.05)$.

Conclusions Empiric antibiotic de-escalation appears feasible as a routine form of treatment for EOS in late preterm and term neonates.
\end{abstract}

\section{Introduction}

According to the American College of Obstetricians and Gynecologists, group B Streptococcus (GBS) prevention guidelines, late preterm and term deliveries accounted for nearly $80 \%$ of early onset sepsis (EOS) cases [1,2]. Management of suspected EOS in late preterm and term neonates varies widely. In currently published guidelines from medical bodies such as the Canadian Paediatric Society, the American Academy for Pediatrics, the National Institute for

Noraida Mohamed Shah

noraida_mshah@ukm.edu.my

1 Centre for Quality Management of Medicine, Faculty of Pharmacy, Universiti Kebangsaan Malaysia, Jalan Raja Muda Abdul Aziz, 50300 Kuala Lumpur, Malaysia

2 Department of Pediatrics, Hospital Shah Alam, Persiaran Kayangan, Seksyen 7, 40000 Shah Alam, Selangor, Malaysia

\section{Key Points}

Empiric antibiotic de-escalation should be encouraged, especially in cases with low suspicion of early onset sepsis (EOS).

Empiric antibiotic de-escalation can be routinely implemented for EOS because of its comparable treatment outcomes to those in neonates without antibiotic de-escalation.

To prevent unnecessary and prolonged antibiotic use, factors associated with antibiotic de-escalation may be used to determine whether antibiotic use should be deescalated. 
Health and Care Excellence, the Swiss Society of Neonatology, and the Belgian and Flemish Societies of Neonatology and Paediatrics, there is a lack of consensus regarding the best time to review empiric antibiotic treatment for EOS [3]. In Malaysia, disagreements exist between the 4th edition of Paediatric Protocols and 3rd edition of the National Antibiotic Guideline (NAG), with the time recommended to review empiric treatment in EOS listed as $48-72 \mathrm{~h}$ and $36 \mathrm{~h}$, respectively [4-6].

Limited rapid and reliable laboratory sensitivity and specificity, coupled with the non-specific clinical manifestations of EOS pose a challenge regarding the confirmation of an initial diagnosis of sepsis in neonates [7]. Blood culture proof remains the 'gold standard' to diagnose EOS. However, false negatives resulting from blood culture are common among neonates. In previous studies, as few as 5\% of treated suspected EOS cases reported a positive blood culture, while $95 \%$ of EOS cases were treated without evidence of infection [7, 8]. It is now an acceptable practice for pediatricians to treat EOS in spite of a negative blood culture [9].

Selection of empiric antibiotic therapy should take local microbial susceptibility patterns into consideration [10-12]. In cases with no obvious infection or when blood cultures suggest infection is unlikely, antibiotic de-escalation either by discontinuation or narrowing down the antibiotic spectrum is recommended [13-15]. Such action should be taken within $72 \mathrm{~h}$ or as soon as blood culture results are found to be negative $[4,15]$.

De-escalating continued empiric antibiotic use decreases exposure to antibiotics, consequently preventing resistance development and minimizing cost [15]. However, clinical outcomes resulting from antibiotic de-escalation must be at least comparable to the results from the conventional approach of maintaining initial therapy [15]. De-escalation practices have shown favorable clinical outcomes in adult critical care patients with pneumonia, intra-abdominal infections, and septic shock [13-15]. However, current information on de-escalation of empiric antibiotic use in neonates has been limited or not well described.

In suspected EOS, confirmation of infection by positive blood culture is rarely reported $[7,9]$. Therefore, de-escalation of antibiotics within $72 \mathrm{~h}$ of initiation remains difficult to achieve for pediatricians. Historically, decision making has been loosely based on blood culture and mostly guided by the pediatrician's judgment [6]. However, it is possible to de-escalate antibiotics in suspected EOS cases, especially in well-appearing neonates with negative blood culture [16].

In a 2014 randomized controlled trial conducted by Pasha et al., neonates of gestational age (GA) $\geq 34$ weeks with suspected EOS and a negative blood culture were randomized into 3-day and 5-day treatment groups. There were no differences in treatment failure between groups. Treatment failure was defined as the reappearance of sepsis 2 weeks after the discontinuation of treatment [17].

Cordero and Ayers (2003) conducted a study on extremely low birth weight (BW) neonates with an average GA of 25-27 weeks. They found that discontinuation of empiric antibiotics when blood culture was negative for $\leq 3$ days versus continued for $\geq 7$ days resulted in desirable treatment outcomes. Furthermore, mortality was not compromised [18]. Therefore, empiric antibiotic de-escalation in suspected EOS can potentially be implemented, despite wide variations in neonatal GA. Further investigations into this area are needed, including a compilation of evidence from our local setting.

The purpose of this study was to compare characteristics and treatment outcomes between neonates under empiric antibiotic de-escalation therapy and neonates without empiric antibiotic de-escalation. Factors associated with empiric antibiotic de-escalation practice in late preterm and term neonates suspected with EOS were also investigated.

\section{Methods}

This was a prospective observational cohort study conducted at the neonatal intensive care units (NICUs) of two specialist public hospitals in Malaysia. Both NICUs have similar newborn admission rates and were following criteria in the Paediatric Protocols (Ministry of Health, Malaysia) [4] for the management of suspected EOS. This is with regards to the initiation of treatment and de-escalation of empiric antibiotic, which was evaluated based on risk factors, laboratory findings, and clinical progress. These NICUs have full-time clinical pharmacists who actively participate in the drug management in neonates. No intervention or interruption of management by the researcher occurred during the study period. To minimize the Hawthorne effect, all frontline practitioners were not informed about the details of the study, including the study design and types of data collected.

Cases were deemed eligible for inclusion if the neonates were born at a GA greater than 34 weeks and were admitted to the NICU with suspected EOS and started with empiric antibiotic within $72 \mathrm{~h}$ of life. Neonates who never received empiric antibiotic, received escalated empiric antibiotic within $72 \mathrm{~h}$ of treatment, or those who spent less than $72 \mathrm{~h}$ in the NICU were excluded. Ethics approval was obtained from the Medical Research and Ethics Committee, Ministry of Health, Malaysia [NMRR-17-1882-36914 (IIR)].

The medical records of all cases admitted within $72 \mathrm{~h}$ of life in the two NICUs were prospectively screened from 1 September until 31 December 2017 (hospital A) and 1 January until 30 April 2018 (hospital B). Hospital A and B were public specialist hospitals located in Klang Valley, Malaysia, 
and have a similar NICU admission rate per month. Data on eligible cases were collected from patients' electronic and manual medical records. The data included patients' demographic profiles, risk factors (prolonged rupture of membrane $>18 \mathrm{~h}$, maternal pyrexia $>38{ }^{\circ} \mathrm{C}$, maternal high vaginal swab/urine culture positive, history of being a GBS carrier, meconium-stained amniotic fluid, chorioamnionitis, and perinatal asphyxia), clinical manifestations, prescribed antibiotics, types of organisms, de-escalation practices, and treatment outcomes up to 7 days and 28 days of life. Microbiological analyses included blood cultures prior to the commencement of empiric antibiotics.

A late preterm was defined as born between gestational weeks 34 and 36, and term was defined as born at GA 37 weeks and later [19, 20]. Empiric antibiotic de-escalation is defined as narrowing of the antibiotic spectrum by reducing the number of first-line antibiotic combinations (e.g., penicillin/ampicillin plus gentamicin/cefotaxime) or discontinued use of all antibiotics in the absence of any obvious infection within $72 \mathrm{~h}$ of therapy $[13,14]$. Non-de-escalation is defined as the continuation of first-line empiric antibiotics, especially following negative culture results, based on risk assessment [21]. Treatment failure is defined as the need for late antibiotic escalation after $72 \mathrm{~h}$ of empiric antibiotic therapy, strong suspicion of second infection within 7 days of life, or mortality within 7 days of life due to sepsis [22].

Data were stratified according to de-escalation and non-de-escalation practices and were analyzed using IBM $^{\circledR}$ SPSS $^{\circledR}$ for Windows Version 23. The frequencies and percentages of each continuous variable studied were presented in the form of a table. For practice comparison, categorical variables were assessed using Pearson Chi-squared test $\left(\chi^{2}\right.$ test) or Fisher's exact test. Continuous variables were assessed using the Mann-Whitney $U$ test, as the medians from both groups were compared. For all statistical analyses, the significance was set at $p<0.05$. Simple logistic regression was conducted to identify the possible predictors related to de-escalation practice, and variables with $p$ value of $<0.1$ were considered for inclusion in a multiple logistic regression analysis using a forward LR method [23].

\section{Results}

Figure 1 describes the flowchart for the case selection process. A total of 1045 neonates were screened, and 440 neonates fulfilled the inclusion criteria (hospital $\mathrm{A}=172$, hospital $B=268$ ). However, only 429 neonates were included for analysis, as 11 neonates were excluded due to transfer out from the NICU $(n=6)$ and antibiotic escalation $(n=5)$ within $72 \mathrm{~h}$ of treatment. A total of 207 cases were in the deescalation group (106 cases off one antibiotic and 101 cases off all antibiotics within $72 \mathrm{~h}$ ), and 222 cases were under the non-de-escalation group.

The demographic and clinical data of cases were compared (Table 1). Births via cesarean sections, exposure to antenatal steroids, and congenital anomalies were all statistically significantly higher in the de-escalation group versus
Fig. 1 Flowchart of the selection process for cases within $72 \mathrm{~h}$ of empiric antibiotic therapy. $G A$ gestational age

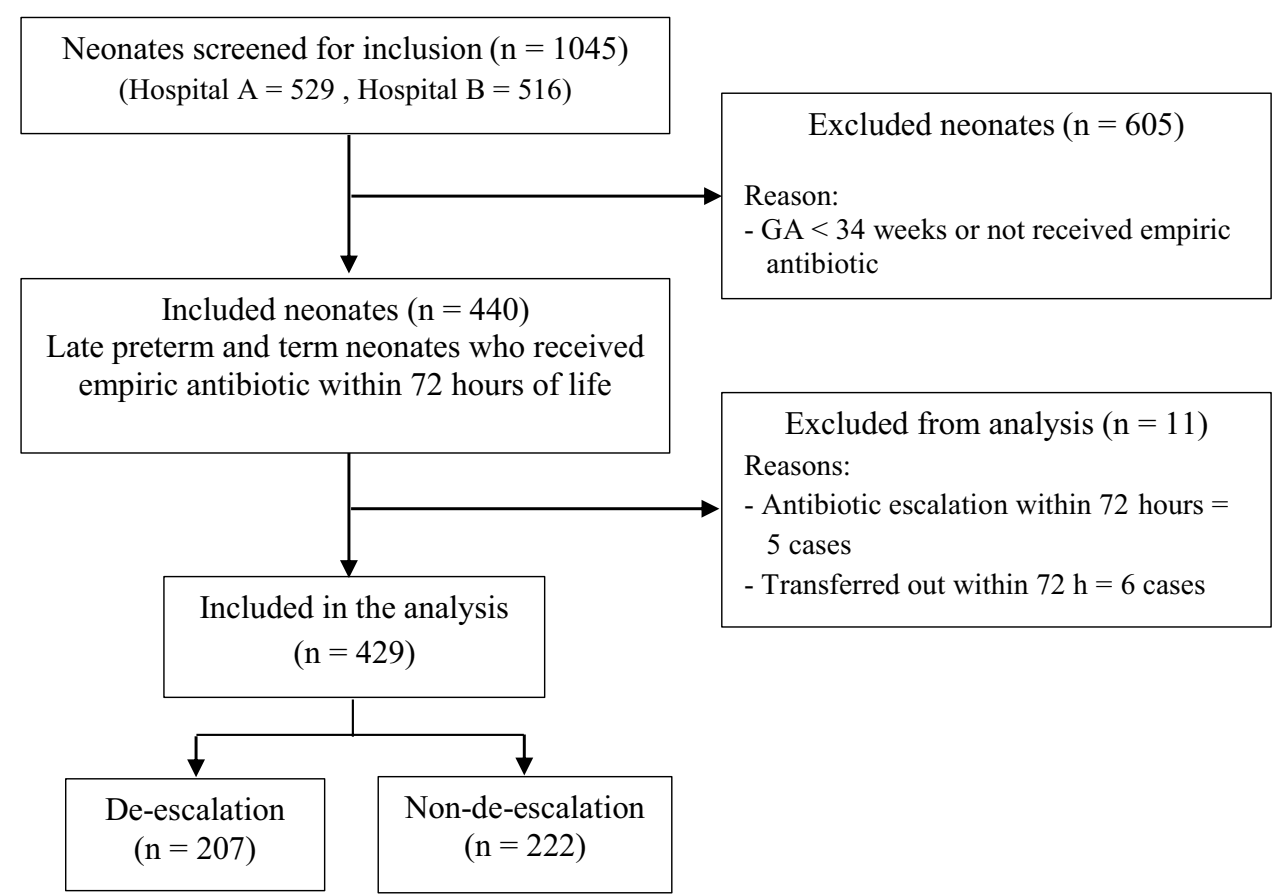




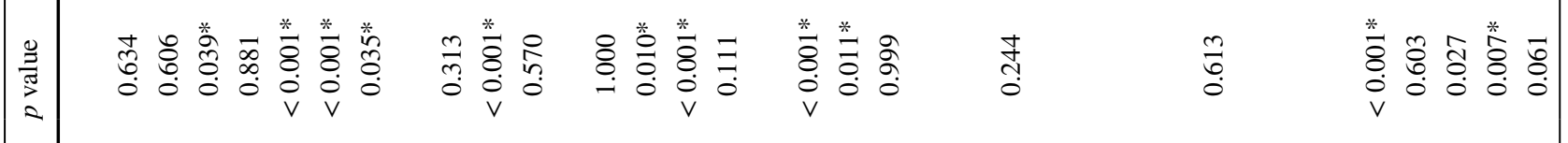

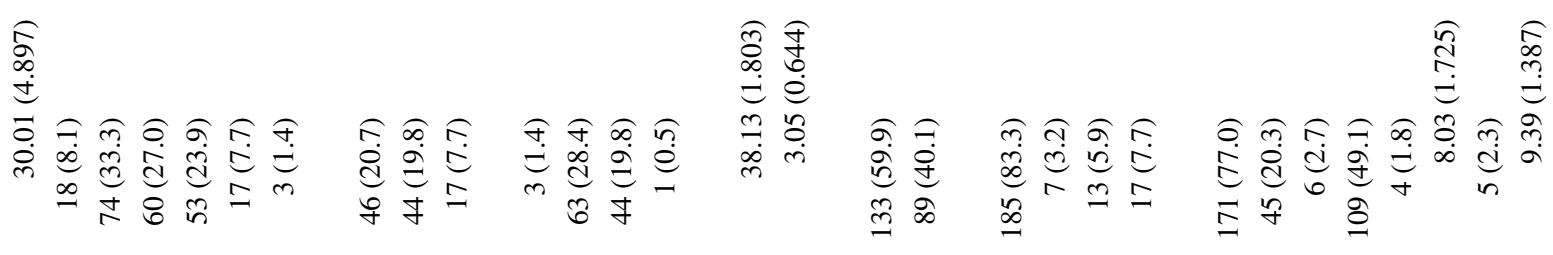

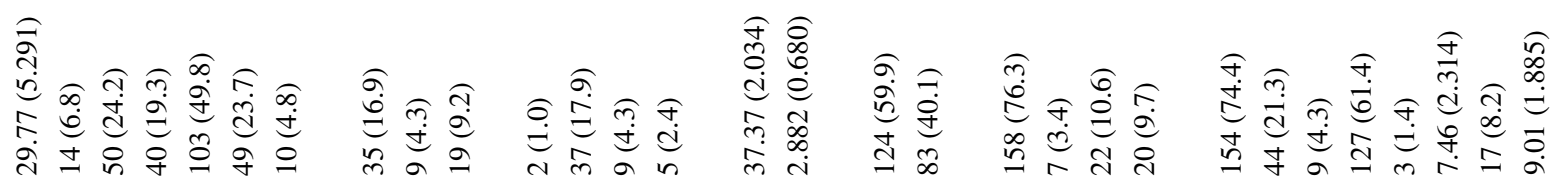
兽

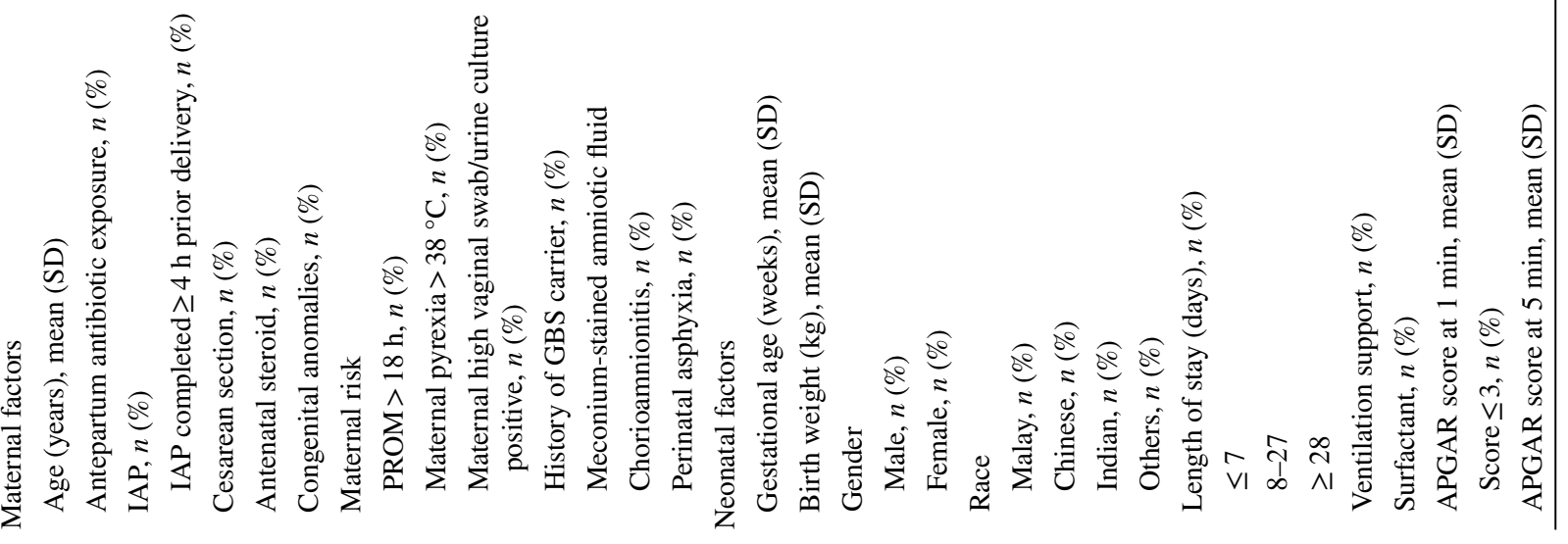




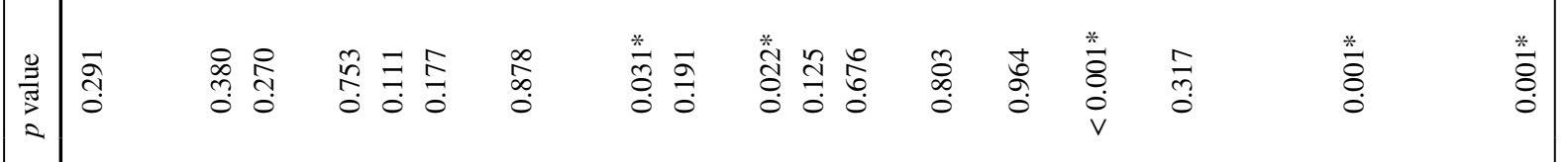

สิ

II

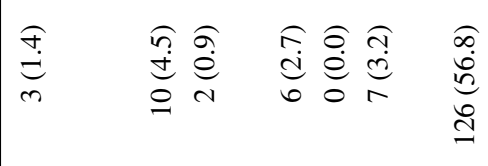

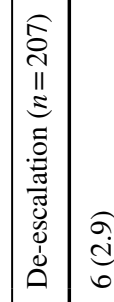

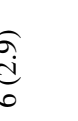

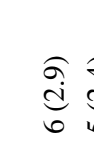

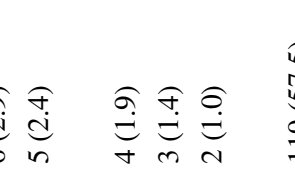

$n$
$\frac{n}{5}$
3

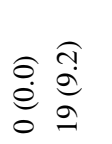

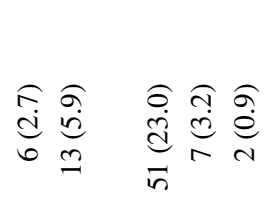

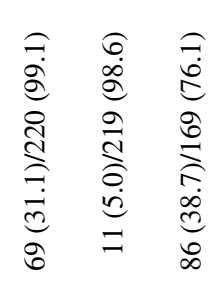

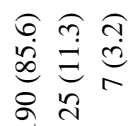

กิ

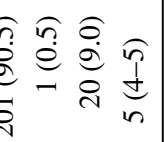

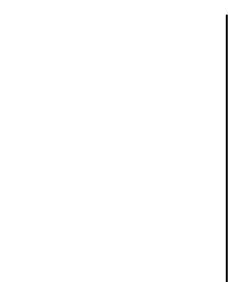

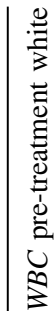

.5

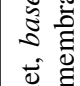

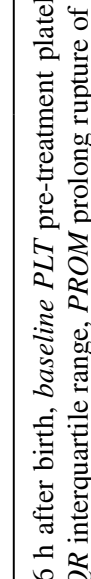

๘。

言高

氜

흉

ํㅜㄹ

.

范

$\approx$

范

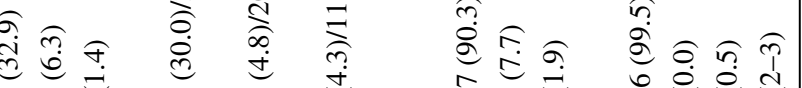

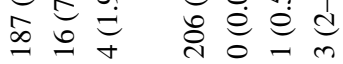
$\infty \underset{0}{\infty}=$

तु - + त०

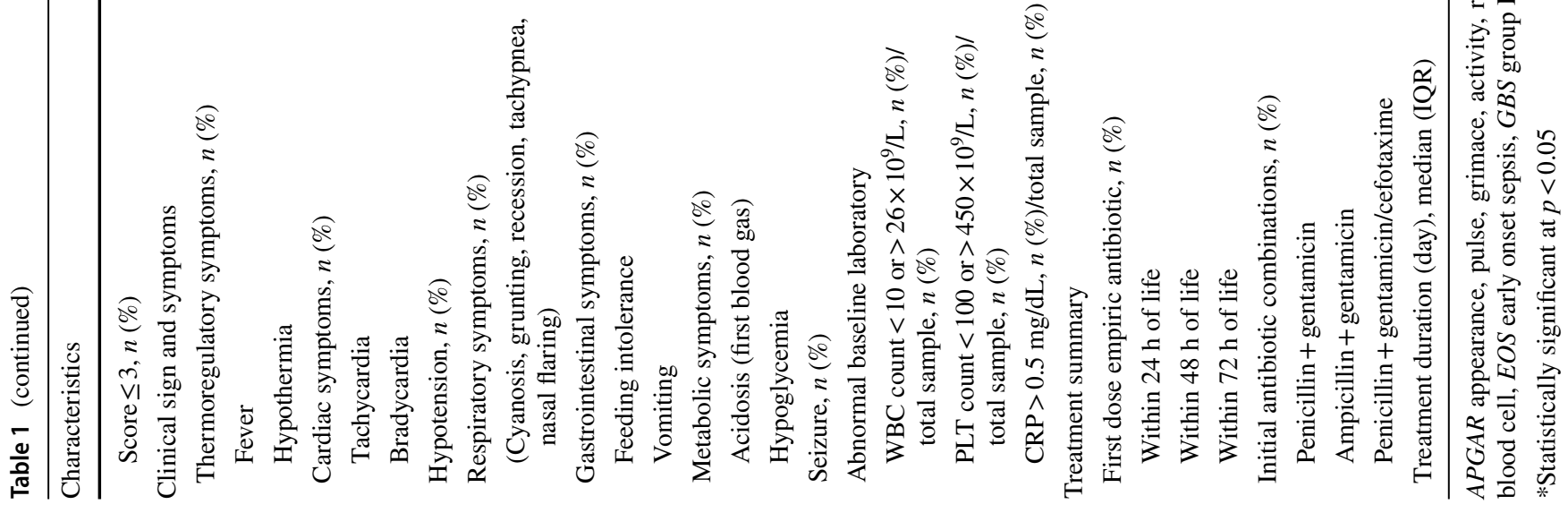


the non-de-escalation group. Intrapartum antibiotic prophylaxis (IAP), maternal pyrexia, meconium-stained amniotic fluid, and chorioamnionitis were significantly higher in the non-de-escalation group versus the de-escalation group. Cases in the de-escalation group had significantly smaller mean GA and BW. However, a significantly higher number of cases in the de-escalation group required early ventilator support and presented with poor Appearance, Pulse, Grimace, Activity, Respiration (APGAR) score at $1 \mathrm{~min}, \leq 3$.

There was no significant difference in the timing of the first dose of empiric antibiotics for both groups. However, there was significant difference in the choice of antibiotics between the groups. The number of cases requiring a switch from gentamicin to cefotaxime was higher in the non-de-escalation group, and this was mostly documented as being for central nervous system antibiotic coverage or due to a suspicion of acute renal impairment. The duration of antibiotic treatment was significantly longer in the nonde-escalation group (Table 1).

Isolated pathogens in blood samples were reported in seven cases (1.6\%). Of these cases, the causative organism was gram positive in five cases. Three of the pathogens were responsible for clinically significant infections with raised C-reactive protein (CRP) $>0.5 \mathrm{mg} / \mathrm{dL}$ (GBS, Listeria monocytogenes, and Klebsiella sp.), and other isolated pathogens were labeled as 'contaminant sample' in the pathology report. Antibiotic sensitivity showed Klebsiella sp. and Sphingomonas (pseudo.) paucimobilis were sensitive to gentamicin, while L. monocytogenes was sensitive to penicillin (Table 2).

Table 3 shows the treatment outcomes up to 7 days and 28 days of life. There was no significant difference in treatment failure levels between both groups. The cause of mortality within 7 days of life was not stated as sepsis (two cases of persistent pulmonary hypertension of the newborn and one case of hydrops fetalis) and therefore was not categorized as treatment failure. Treatment outcomes up to 28 days of life included a high number of suspicions of second infection and mortality after 7 days of life in de-escalation group versus the non-de-escalation group. However, the difference was not statistically significant.

Identified factors with a $p<0.1$ based on univariate analysis were exposure to IAP, cesarean section delivery, exposure to antenatal steroids, congenital anomalies, maternal pyrexia, meconium-stained amniotic fluid, chorioamnionitis, GA, BW, ventilator support, surfactant, poor APGAR score at $1 \mathrm{~min}$, acidosis, and CRP $>0.5 \mathrm{mg} / \mathrm{dL}$. The identified factors were included in the multivariate analysis. Based on the multivariate analysis model, cesarean section delivery, exposure to antenatal steroids, nil history of maternal pyrexia,

Table 2 Clinical characteristics of neonates showing causative organism isolated in blood sample $(n=7)$

\begin{tabular}{|c|c|c|c|c|c|}
\hline Case & GA; BW & Pathogens; laboratory & EOS risk factors & Reason for EOS evaluation & Treatment description \\
\hline \multicolumn{6}{|c|}{ De-escalation } \\
\hline 1 & $39 ; 3.16$ & $\begin{array}{l}\text { Cellulomonas sp.; WBC 15.3, } \\
\text { PLT 280, CRP } 0.02\end{array}$ & PROM $>18 \mathrm{~h}$ & $\begin{array}{l}\text { Well-appearing neonate, evalu- } \\
\text { ated because of risk factors }\end{array}$ & $\begin{array}{l}\text { Gentamicin } 2 \text { doses } \\
\text { Penicillin } 10 \text { doses } \\
\text { Treatment duration: } 5 \text { days }\end{array}$ \\
\hline 2 & $39 ; 2.9$ & $\begin{array}{l}\text { Group B Streptococcus; WBC } \\
\text { 4.4, PLT 214, CRP } 7.9\end{array}$ & $\begin{array}{l}\text { Meconium-stained amniotic } \\
\text { fluid }\end{array}$ & $\begin{array}{l}\text { Respiratory distress at birth } \\
\text { with tachycardia }\end{array}$ & $\begin{array}{l}\text { Gentamicin } 2 \text { doses } \\
\text { Penicillin } 20 \text { doses } \\
\text { Treatment duration: } 10 \text { days }\end{array}$ \\
\hline 3 & $36 ; 2.23$ & $\begin{array}{l}\text { Bacillus sp.; WBC 16.2, PLT } \\
\text { 275, CRP } 0.02\end{array}$ & Premature with low BW & Hypoglycemia & $\begin{array}{l}\text { Gentamicin } 2 \text { doses } \\
\text { Penicillin } 10 \text { doses } \\
\text { Treatment duration: } 5 \text { days }\end{array}$ \\
\hline 4 & $37 ; 3.24$ & $\begin{array}{l}\text { Sphingomonas (pseudo.) } \\
\text { paucimobilis; WBC 27.3, } \\
\text { PLT } 218, \text { CRP } 0.02\end{array}$ & None & Respiratory distress at birth & $\begin{array}{l}\text { Gentamicin } 2 \text { doses } \\
\text { Penicillin } 14 \text { doses } \\
\text { Treatment duration: } 7 \text { days }\end{array}$ \\
\hline 5 & $37 ; 3.87$ & $\begin{array}{l}\text { Staphylococcus, coagulase } \\
\text { negative; WBC 25.9, PLT } \\
268, \text { CRP } 0.06\end{array}$ & None & Respiratory distress at birth & $\begin{array}{l}\text { Gentamicin } 2 \text { doses } \\
\text { Penicillin } 10 \text { doses } \\
\text { Treatment duration: } 5 \text { days }\end{array}$ \\
\hline \multicolumn{6}{|c|}{ Non-de-escalation } \\
\hline 6 & $35 ; 2.28$ & $\begin{array}{l}\text { Listeria monocytogenes; WBC } \\
\text { 20.7, PLT 199, CRP } 3.8\end{array}$ & $\begin{array}{l}\text { Meconium-stained amniotic } \\
\text { fluid }\end{array}$ & $\begin{array}{l}\text { Respiratory distress with } \\
\text { acidosis }\end{array}$ & $\begin{array}{l}\text { Gentamicin } 6 \text { doses } \\
\text { Penicillin } 18 \text { doses } \\
\text { Treatment duration: } 9 \text { days }\end{array}$ \\
\hline 7 & $39 ; 3.4$ & $\begin{array}{l}\text { Klebsiella sp.; WBC 16.3, PLT } \\
\text { 160, CRP } 10.29\end{array}$ & $\begin{array}{l}\text { PROM }>18 \mathrm{~h} \\
\text { Positive maternal culture }\end{array}$ & $\begin{array}{l}\text { Well-appearing neonate, evalu- } \\
\text { ated because of risk factors }\end{array}$ & $\begin{array}{l}\text { Gentamicin } 1 \text { dose Cefo- } \\
\text { taxime } 14 \text { doses } \\
\text { Penicillin } 19 \text { doses } \\
\text { Treatment duration: } 6 \text { days }\end{array}$ \\
\hline
\end{tabular}

$B W$ birth weight, $C R P$ C-reactive protein, EOS early onset neonatal sepsis, GA gestational age, $P L T$ platelet, $P R O M$ prolong rupture of membrane, $W B C$ white blood cell 
Table 3 Treatment outcomes with antibiotic use for EOS $(n=429)$

\begin{tabular}{lcc}
\hline & De-escalation $(n=207)$ & Non-de-escalation $(n=222)$ \\
\hline Outcomes up to 7 days of life & & $12(5.4)$ \\
Treatment failure, $n(\%)$ & $14(6.8)$ & $10(4.5)$ \\
Antibiotic escalation after $72 \mathrm{~h}, n(\%)$ & $7(3.4)$ & $2(0.9)$ \\
Suspicion of second infection within 7 days of life, $n(\%)$ & $7(3.4)$ & $1(0.5)$ \\
Mortality within 7 days of life, $n$ & $2(1.0)$ & $3(1.4)$ \\
Outcomes up to 28 days of life & & $213(95.9)$ \\
Status at the end of follow-up & $4(1.9)$ & $3(1.4)$ \\
Awaiting growth, $n(\%)$ & $191(92.3)$ & $2(0.9)$ \\
Survived to discharge, $n(\%)$ & $8(3.9)$ & $4(1.8)$ \\
Suspicion of second infection after 7 days of life, $n(\%)$ & $6(2.9)$ & 0.359 \\
Mortality, $n(\%)$ & $6(2.9)$ & \\
Others, $n(\%)$ & & \\
\hline
\end{tabular}

EOS early onset sepsis

Table 4 Factors associated with antibiotic de-escalation practice among late preterm and term neonates in suspected EOS

\begin{tabular}{llllr}
\hline Variables & Crude OR $(95 \% \mathrm{CI})^{\mathrm{a}}$ & Beta & ${\text { Adjusted OR }(95 \% \mathrm{CI})^{\mathrm{b}}}^{p}$ value $^{\mathrm{b}}$ \\
\hline Cesarean section delivery & $3.19(2.11-4.82)$ & 1.27 & $3.54(1.93-6.51)$ & $<0.001^{*}$ \\
Antenatal steroids & $3.75(2.10-6.75)$ & 0.87 & $2.40(1.04-5.55)$ & $0.041^{*}$ \\
No maternal pyrexia $>38^{\circ} \mathrm{C}$ & $5.44(2.58-11.46)$ & 1.35 & $3.85(1.55-9.60)$ & $0.004^{*}$ \\
No meconium-stained amniotic fluid & $1.82(1.15-2.88)$ & 0.74 & $2.10(1.07-4.12)$ & $0.030^{*}$ \\
Normal baseline C-reactive protein $\leq 0.5 \mathrm{mg} / \mathrm{dL}$ & $7.38(3.91-13.95)$ & 2.14 & $8.51(4.23-17.15)$ & $<0.001^{*}$ \\
\hline
\end{tabular}

$C I$ confidence interval, EOS early onset sepsis, $O R$ odds ratio

*Statistically significant at $p<0.05$

${ }^{\text {a }}$ Simple logistic regression

${ }^{\mathrm{b}}$ Multiple logistic regression

absence of meconium-stained amniotic fluid, and normal baseline $\mathrm{CRP} \leq 0.5 \mathrm{mg} / \mathrm{dL}$ were factors significantly associated with de-escalation practice (Table 4).

\section{Discussion}

In spite of the low incidence of culture-proven infection, the continued overuse of antibiotics to treat late preterm and term neonates with suspected EOS drove the desire to evaluate such a practice in our local setting [3]. In this study, we found that slightly over $40 \%$ of total admitted neonates were started on empiric antibiotics. Nearly half of them underwent empiric antibiotic de-escalation within $72 \mathrm{~h}$, while the remaining patients continued treatment beyond $72 \mathrm{~h}$. Antibiotic de-escalation was not protocolized practice and was performed by the physician in charge of the patient in accordance with the patient's clinical evolution and other investigations [21]. Thus, the de-escalation rate may vary between hospitals. Currently, no study has reported on the antibiotic de-escalation rate among neonates suspected with
EOS. However, in adults, the antibiotic de-escalation rate was reported to be $51 \%$ in generally ill patients and ranging from 6 to $74 \%$ in ventilator-associated pneumonia patients [21].

In this study, antibiotic practice was guided by the National Antibiotic Guidelines (2019). According to this guideline, in the management of suspected EOS, if blood culture is negative and initial clinical suspicion is not strong, antibiotic de-escalation should be considered at $48 \mathrm{~h}$ of treatment after reassuring neonate condition with low CRP. However, if blood culture is positive or there is a strong clinical suspicion of sepsis with a negative blood culture, treatment may be given up to 5-7 days [5].

This study showed similar characteristics for the neonates in both groups (de-escalation and non-de-escalation). These include average GA $>37$ weeks, normal $\mathrm{BW}>2.5 \mathrm{~kg}$, and similar gender and race distributions, with no significant difference in length of hospital stay. Respiratory-related symptoms such as cyanosis, grunting, recession, tachypnea, and nasal flaring were the most common manifestations documented in both groups. However, clinical manifestations 
alone were too non-specific to determine true infections in EOS. Further clinical investigations were needed to confirm EOS $[1,7]$.

In this study, the majority of suspected EOS cases were started on empiric antibiotic penicillin plus gentamicin within $24 \mathrm{~h}$, in concordance with the national guidelines $[4,5]$. To reduce the risks of mortality and morbidity, once EOS is suspected, it is crucial to start empiric antibiotics immediately [24-26]. A higher number of cases requiring change of antibiotic regime from penicillin plus gentamicin to penicillin plus cefotaxime was observed in the non-deescalation group in our study.

Cefotaxime is a third-generation cephalosporin and is listed as an alternative first-line antibiotic in the NAG [5]. Most of the changes were due to suspected acute renal impairment, which can be worsened by gentamicin administration. However, studies have shown that gentamicin's nephrotoxic effect is less frequent in neonates than in adults [27]. Severe cases of EOS require antibiotics with extensive coverage and excellent cerebrospinal fluid penetration properties. Therefore, cefotaxime is an antibiotic of choice. However, cefotaxime use should be restricted due to rapid development of resistance and risk of invasive candidiasis if routinely used for the treatment of EOS [1]. In this study, less than $5 \%$ of the total neonates analyzed were exposed to cefotaxime and none of them developed resistance or candidiasis during their NICU stay.

Besides that, neonates in the non-de-escalation group also reported significantly longer durations of antibiotic use. However, previous studies reported that shorter treatment duration in suspected EOS did not increase the risk of late-onset sepsis, treatment failure, necrotizing enterocolitis or mortality $[17,18,28]$. Similarly, no difference in the frequency of treatment failure was observed in our study.

Overall, positive blood culture was reported in less than $2 \%$ of total cases in our study. Furthermore, five out of seven pathogens isolated were gram-positive organisms. These results are in line with those obtained from previous studies conducted in Malaysia, Estonia, and the United States, in which positive blood culture was obtained in $<5 \%$ of the study population, was rarely reported, and around $70 \%$ of causative pathogens were gram positive [29-33]. False-negative blood culture is common in the neonatal population, possibly due to insufficient blood sample volumes [34] or low-density bacteremia due to perinatal antibiotic exposure [35]. As a result, treating EOS in the presence of a negative blood culture remains the normal practice [9, 36, 37]. However, using blood culture to confirm the diagnosis of EOS remains the 'gold standard.' In the future, more advanced methods such as polymerase chain reaction (PCR) may be useful to improve the EOS diagnosis sensitivity [38].

In our final model, five predictors were identified to be associated with antibiotic de-escalation within $72 \mathrm{~h}$ of therapy. They are cesarean section delivery, exposure to antenatal steroids, nil history of maternal pyrexia, absence of meconium-stained amniotic fluid, and normal baseline CRP. A cesarean section delivery was classified as a cleancontaminated operation, with a risk of infection and endometritis to the operative mother, but no additional risk of EOS to the neonate, since there was no contact with the birth canal during the delivery process [39, 40]. Exposure to antenatal steroids was also a predictive indicator for deescalation practice. To accelerate fetal lung maturation and improve neonatal respiratory function upon delivery, antenatal steroids are given to mothers at risk of premature delivery or elective cesarean before 38 weeks gestation [41]. A study by Gyamfi-Bannerman et al. (2016) at the Maternal-Fetal Medicine Units Network of the Eunice Kennedy Shriver National Institute of Child Health and Human Development reported that completing two doses of antenatal steroids was not associated with an increased risk of EOS [42].

In this study, there were two maternal risk factors associated with antibiotic de-escalation practice. They are nil history of maternal pyrexia and absence of meconium-stained amniotic fluid. Maternal pyrexia is a risk factor listed in most guidelines and is a criterion to start empiric antibiotic therapy [16, 43]. Nonetheless, previous studies reported that only about $6.4 \%$ of evaluated neonates were infected when the maternal recorded temperature was $38.9^{\circ} \mathrm{C}$, with reduced risk as temperature drops [16, 43, 44]. Meconiumstained amniotic fluid was not considered as a criterion to start empiric antibiotics, as meconium aspiration syndrome presented in only $5 \%$ of meconium-stained amniotic fluid cases $[45,46]$. Therefore, if neonates are clinically well without risks of maternal pyrexia and meconium-stained amniotic fluid, empiric antibiotics de-escalation can safely be implemented.

A normal baseline level of CRP was identified as a predictor for antibiotics de-escalation in our study. It is a widely used biomarker with a high specificity. However, it has a low sensitivity for indicating neonatal sepsis, as its levels rise in non-infectious events as well [47, 48]. CRP concentration may appear normal in the early stage of infection, and it has limited predictive value in the single figures, due to physiological variations in the first few days of life [7]. Serial CRP in the first 24-48 h of symptoms increases test sensitivity and can be helpful in determining the duration of empiric antibiotic use [7, 16, 49, 50]. However, in this study, serial CRP to guide treatment decisions was not a routine practice.

This study had some limitations because de-escalation or non-de-escalation practices were based on clinician preference and involved only two public hospitals. Therefore, results cannot be generalized. Besides, this study did not use the Kaiser risk assessment calculator to determine whether an antibiotic should be started for EOS [51]. Instead, the criteria used was based on our local reference Paediatric 
Protocols (4th edition) as this was the reference commonly used by the physicians at our study sites. Hence, there could be a possibility that an antibiotic was started even though the EOS risk was low according to the Kaiser risk assessment. It is not surprising that most of the identified factors in this study are factors currently included in the Kaiser risk assessment. Despite the limitations, to our knowledge, this is the first local study investigating factors associated with empiric antibiotic de-escalation among neonates with suspected EOS.

In conclusion, this study demonstrated that among late preterm and term neonates suspected with EOS, strategic empiric antibiotic de-escalation was possible in most cases, without any negative impact on clinical outcomes.

Acknowledgements We (the authors) would like to thank the DirectorGeneral of Health Malaysia for the permission to publish this paper. We are grateful to Dr. See Kwee Ching, Consultant Neonatologist of Sungai Buloh Hospital and Head of Pediatric Department Hospital Shah Alam for their support toward this research project. We are also thankful to Faculty of Pharmacy Universiti Kebangsaan Malaysia, Pharmacy Department and Neonatal Intensive Care Unit of Sungai Buloh Hospital and Hospital Shah Alam for their technical support.

\section{Compliance with Ethical Standards}

Funding None.

Conflict of interest The authors, Nazedah Ain Ibrahim, Mohd Makmor Bakry, Nurul Ain Mohd Tahir, Nur Rashidah Mohd Zaini, and Noraida Mohamed Shah, declare that they have no conflicts of interest.

\section{References}

1. Polin RA. Management of neonates with suspected or proven early-onset bacterial sepsis guidance for the clinician in rendering pediatric care. Pediatrics. 2012;129:1006-15.

2. Committee opinion: prevention of early-onset group B streptococcal disease in newborns. The American College of Obstetricians and Gynecologist (ACOG). 2011.

3. van Herk W, el Helou S, Janota J, Hagmann C, Klingenberg C, Staub E, Giannoni E, et al. Variation in current management of term and late-preterm neonates at risk for early-onset sepsis. An international survey and review of guidelines. Pediatr Infect Dis J. 2016;35(5):494-500.

4. Paediatric Protocols. 4th ed. Malaysia: Ministry of Health; 2018.

5. National Antibiotic Guideline. 3rd ed. Malaysia: Ministry of Health; 2019.

6. Ibrahim NA, Bakry MM, Ishak S, Shah NM. A review of antibiotic used in suspected early-onset neonatal sepsis from Malaysian perspective: Which ones to choose and how long to give? Asian J Pharm Clin Res. 2019;12(1):529-36.

7. Bedford RAR, Kumar R. Early onset neonatal sepsis: diagnostic dilemmas and practical management. Arch Dis Child Fetal Neonatal Ed. 2014;0:F1-F5.

8. Cantey JB, Wozniak PS, Sánchez PJ. Prospective surveillance of antibiotic use in the neonatal intensive care unit: results from the SCOUT study. Pediatr Infect Dis J. 2015;34:267-72.
9. Cantey JB, Baird SD. Ending the culture of culture-negative sepsis in the neonatal ICU. Pediatrics. 2017;140(4):e20170044.

10. Mukhopadhyay S, Eichenwald EC, Puopolo KM. Neonatal earlyonset sepsis evaluations among well-appearing infants: projected impact of changes in CDC GBS guidelines. J Perinatol. 2013;33:198-205.

11. Eskandarian N, Pillay SG, Ismail Z, Neela VK. Characterization of a Group B Streptococcus infection based on the demographics, serotypes, antimicrobial susceptibility and genotypes of selected isolates from sterile and non-sterile isolation sites in three major hospitals in Malaysia. J Infect Public Health. 2017;10:14-21.

12. Azirah N, Sani M, Sapri HF, Neoh H, Hussin S. First report on the molecular epidemiology of Malaysian Staphylococcus epidermidis isolated from a University Teaching Hospital. BMC Res Notes. 2014;7:597.

13. Masterton RG. Antibiotic de-escalation. Crit Care Clin. 2011:27:149-62.

14. Garnacho-Mentero J, Escoresca-Ortega A, Fernandez-Delgado E. Antibiotic de-escalation in the ICU: how is it best done? Curr Opin Infect Dis. 2015;28:193-8.

15. Camargo LFA. The "de-escalation concept"' and antibiotic deescalation: a missed opportunity? Shock. 2013;39(7):29-31.

16. Jefferies AL. Management of term infants at increased risk for early-onset bacterial sepsis; Canadian Paediatric Society, Fetus and Newborn Committee, Ottawa, Ontario. Paediatr Child Health. 2017;223-8.

17. Pasha YZ, Ahmadpour-Kacho M, Behmadi R, Jahangir T. 3-day versus 5-day course of intravenous antibiotics for suspected early onset neonatal sepsis: a randomized controlled trial. Iran J Pediatr. 2014;24(6):673-8.

18. Cordero L, Ayers LW. Duration of empiric antibiotics for suspected early onset sepsis in extremely low birth weight infants. Infect Control Hosp Epidemiol. 2003;24(9):662-6.

19. Cohen-Wolkowiez M, Moran C, Benjamin DK, Cotton CM, Clark $\mathrm{RH}$, Benjamin DK Jr, et al. Early and late onset sepsis in late preterm infants. Pediatr Infect Dis J. 2009;28(12):1052-6.

20. Wynn JL, Wong HR, Shanley TP, Bizzarro MJ, Saiman L, Polin RA. Time for a neonatal-specific consensus definition for sepsis. Pediatr Crit Care Med. 2014;15(6):523-8.

21. Gonzalez L, Cravoisy A, Barraud D, Conrad M, Nace L, Lemarie $\mathrm{J}$, et al. Factors influencing the implementation of antibiotic deescalation and impact of this strategy in critically ill patients. Crit Care. 2013;17:R140.

22. Metsvaht T, Pisarev H, Ilmoja M-L, Parm U, Maipuu L, Merila M, et al. Clinical parameters predicting failure of empirical antibacterial therapy in early onset neonatal sepsis, identified by classification and regression tree analysis. BioMed Cent. 2009;72(9):1-11.

23. Woodward M., Epidemiology: study design and data analysis, 3rd ed. London: CRC Press/Chapman and Hall.

24. Fjalstad JW, Stensvold HJ, Bergseng H, Simonsen GS, Salvesen B, Rønnestad AE, et al. Early-onset sepsis and antibiotic exposure in term infants a nationwide population-based study in Norway. Pediatr Infect Dis J. 2016;35:1-6.

25. Benitz WE, Gould JB, Druzin ML. Antimicrobial prevention of early-onset group B streptococcal sepsis: estimates of risk reduction based on a critical literature review. Pediatrics. 1999;103:e78.

26. Duvoisina G, Fischera C, Maucort-Boulch D, Giannonia E. Reduction in the use of diagnostic tests in infants with risk factors for early-onset neonatal sepsis does not delay antibiotic treatment. Swiss Med Wkly. 2014;144:w13981.

27. Hossain MM, Chowdhury NA, Shirin M, Saha SK, Miller-Bell M, Edwards D, et al. Simplified dosing of gentamicin for treatment of sepsis in Bangladeshi neonates. J Health Popul Nutr. 2009;27(5):640-7. 
28. Cotten CM, Taylor S, Stoll B, Goldberg RN, Hansen NI, Sánchez $\mathrm{PJ}$, et al. Prolonged duration of initial empirical antibiotic treatment is associated with increased rates of necrotizing enterocolitis and death for extremely low birth weight infants. Pediatrics. 2009;123:58-66.

29. Ibrahim NA, Manan MM. Early onset neonatal sepsis pathogens in Malaysian hospitals: Determining empiric antibiotic. Int J Pharmacol Pharm Sci. 2014;8(10):703-7.

30. Metsvaht T, Ilmoja M-L, Parm Ü, Maipuu L, Merila M, Lutsar I. Comparison of ampicillin plus gentamicin vs. penicillin plus gentamicin in empiric treatment of neonates at risk of early onset sepsis. Acta Paediatr. 2010;99:665-72.

31. Clark RH, Bloom BT, Spitzer AR, Gerstmann DR. Empiric use of ampicillin and cefotaxime, compared with ampicillin and gentamicin, for neonates at risk for sepsis is associated with an increased risk of neonatal death. Pediatrics. 2006;17:67-74.

32. Boo N, Tunku U, Rahman A, Rohana J. Usefulness of a semiquantitative procalcitonin test kit for early diagnosis of neonatal sepsis. Singap Med J. 2008;49(3):204.

33. Eskandarian N, Neela V, Ismail Z, Puzi SM, Hamat RA, Desa MNM, et al. Group B streptococcal bacteremia in a major teaching hospital in Malaysia: a case series of eighteen patients. Int J Infect Dis. 2013;17(9):e777-80.

34. Haque KN. Definitions of bloodstream infection in the new-born. Pediatr Crit Care Med. 2005;6(3):S45-9.

35. Chiesa C, Pellegrini G, Panero A, Osborn JF, Signore F, Assumma M, Pacifico L. C-reactive protein, interleukin-6, and procalcitonin in the immediate postnatal period: influence of illness severity, risk status, antenatal and perinatal complications, and infection. Clin Chem. 2003;49(1):60-8.

36. Ottolini MC, Lundgren K, Mirkinson LJ, Cason S, Ottolini MG. Utility of complete blood count and blood culture screening to diagnose neonatal sepsis in the asymptomatic at risk newborn. Pediatr Infect Dis J. 2003;22:430-4.

37. Shah BA, Padbury JF. Neonatal sepsis an old problem with new insights. Virulence. 2014;5(1):170-8.

38. Shane AL, Sanchez PJ, Stoll BJ. Neonatal sepsis. Seminar. 2017. https://doi.org/10.1016/S0140-6736(17)31002-4.

39. Gunn B, Ali S, Abdo-Rabbo A, Suleiman B. An investigation into perioperative antibiotic use during lower segment caesarean sections (LSCS) in four hospitals in Oman. Oman Med J. 2009;24:179-83.

40. Signore C, Klebanoff M. Neonatal morbidity and mortality after elective cesarean delivery. Clin Perinatol. 2008;35(2):361-6.

41. Green-top guideline: antenatal corticosteroids to reduce neonatal morbidity and mortality. Royal College of Obstretrician and Gynaecologist (RCOG). 2010.

42. Gyamfi-Bannerman YSC, Thom EA, Blackwell SC, Tita ATN, Reddy UM, Saade GR et al. Antenatal betamethasone for women at risk for late preterm delivery. N Engl J Med. 2016;374(14):1311-20.

43. Mukhopadhyay S, Dukhovny D, Mao W, Eichenwald EC, Puopolo KM. 2010 perinatal GBS prevention guideline and resource utilization. Pediatrics. 2014;133(2):196-203.

44. Escobar GJ. The neonatal 'sepsis work-up': personal reflections on the development of an evidence-based approach toward newborn infections in a managed care organization. J Am Acad Paediatr. 1999:103:360.

45. Gelfand SL, Fanaroff JM, Walsh MC. Meconium stained fluid: approach to the mother and the baby. J Pediatr Clin N Am. 2004;51(3):655-67.

46. Yurdakok M. Meconium aspiration syndrome: do we know? Turk J Pediatr. 2011;53:121-9.

47. Chauhan N, Tiwari S, Jain U. Potential biomarkers for effective screening of neonatal sepsis infections: an overview. Microb Pathog. 2017;107:234-42.

48. Ganesan P, Shanmugam P, Sattar SBA, Shankar SL. Evaluation of IL-6, CRP and hs-CRP as early markers of neonatal sepsis. Clin Diagn Res. 2016;10(5):13-7.

49. Cotten CM, Smith PB. Duration of empirical antibiotic therapy for infants suspected of early-onset sepsis. Curr Opin. 2013;25(2):167-71.

50. Rubin LG, Sánchez PJ, Siegel J, Levine G, Saiman L, Jarvis WR. Evaluation and treatment of neonates with suspected lateonset sepsis: a survey of Neonatologists' practices. Pediatrics. 2002;110:e42.

51. Neonatal early-onset sepsis calculator. https://neonatalsepsiscalcul ator.kaiserpermanente.org. 